\title{
Gradient Theory simulations of pure fluid interfaces using a generalized expression for influence parameters and a Helmholtz energy equation of state for fundamentally consistent two-phase calculations
}

\author{
Rainer N. Dahms* \\ Combustion Research Facility, Sandia National Laboratories, Livermore, CA 94551, USA
}

\begin{abstract}
The fidelity of Gradient Theory simulations depends on the accuracy of saturation properties and influence parameters, and require equations of state (EoS) which exhibit a fundamentally consistent behavior in the two-phase regime. Widely applied multi-parameter EoS, however, are generally invalid inside this region. Hence, they may not be fully suitable for application in concert with Gradient Theory despite their ability to accurately predict saturation properties. The commonly assumed temperature-dependence of pure component influence parameters usually restricts their validity to subcritical temperature regimes. This may distort predictions for general multi-component interfaces where temperatures often exceed the critical temperature of vapor phase components. Then, the calculation of influence parameters is not well defined. In this paper, one of the first studies is presented in which Gradient Theory is combined with a next-generation Helmholtz energy EoS which facilitates fundamentally consistent calculations over the entire two-phase regime. Illustrated on pentafluoroethane as an example, reference simulations using this method are performed. They demonstrate the significance of such high-accuracy and fundamentally consistent calculations for the computation of interfacial properties. These reference simulations are compared to corresponding results from cubic
\end{abstract}

${ }^{*}$ Corresponding author, Rndahms@sandia.gov, +1 (925) 294-6496, P.O. Box 969, MS 9051 
PR EoS, widely-applied in combination with Gradient Theory, and mBWR EoS. The analysis reveals that neither of those two methods succeeds to consistently capture the qualitative distribution of obtained key thermodynamic properties in Gradient Theory. Furthermore, a generalized expression of the pure component influence parameter is presented. This development is informed by its fundamental definition based on the direct correlation function of the homogeneous fluid and by presented high-fidelity simulations of interfacial density profiles. The new model preserves the accuracy of previous temperature-dependent expressions, remains well-defined at supercritical temperatures, and is fully suitable for calculations of general multi-component two-phase interfaces.

Keywords: Gradient Theory, interfacial tension, influence parameter, equation of state, pure fluid, metastability

2010 MSC: 00-01, 99-00

\section{Introduction}

Two-phase interfacial properties such as the surface tension, nucleation, or interfacial thickness represent important physical parameters in a wide variety of technical applications. The strength of surface tension forces significantly

5 affects the dynamical behavior of fluid interfaces. As such, they partly determine, for example, atomization and breakup processes of two-phase flows $[1,2]$ or the chemistry and adhesion of thin films over surfaces $[3,4]$. The significance of such processes for many modern power and propulsion systems such as diesel engines $[5,6,7,8,9,10,11]$, spark-ignition engines $[12,13,14,15$,

$\left.{ }_{10} 16,17,18,19,20,21,22\right]$, or gas turbines $[23,24,25,26,27,28]$ is widely established and well recognized. Nucleation describes the formation of a new thermodynamic phase or structure when the free energy barrier is overcome. Classical nucleation theory $[29,30,31]$ or spinodal decomposition as described by the Cahn-Hilliard equation [32, 33] are classical theories to quantify the dy15 namics of homogeneous nucleation without the presence of preferential sites. 
Nucleation plays a central role in many technical applications such as in polymer and alloy systems. The formation of interfacial regions of finite thicknesses between different homogeneous phases is a consequence of the unstable thermodynamic behavior in certain regimes of fluid densities [32]. The dimensions of vapor-liquid interfaces can have important implications for high-pressure liquid injection processes [34, 35, 36, 37].

Gradient Theory provides a widely accepted methodology to calculate detailed two-phase interfacial properties between gases and liquids [32, 38, 39, $40,41]$. The foundation of this theory was established by van der Waals in $251893[38,39]$ and reformulated later by Cahn and Hilliard in 1958 [32]. It is considered an approximate form of density functional theory [42] where the free energy of a general inhomogeneous system is expressed as a functional of its density profile $[43,44,45]$. While density functional theory utilizes detailed intermolecular potentials, Gradient Theory is facilitated by a mean-field approx-

30 imation of a fundamentally consistent description of isotherms in meta-stable and unstable regimes of fluid densities [32, 46, 47]. Gradient Theory provides a thermo-mechanical model of continuous fluid media that converts statistical mechanics of inhomogeneous fluids into a non-linear boundary value problem. At equilibrium, as applied in this paper, the model has been shown in detail to 35 be equivalent to mean-field molecular theories of capillarity. Recently, Gradient Theory has been successfully compared, notably even for low reduced temperatures, to molecular dynamics/Monte Carlo simulations of vapor-liquid and liquid-liquid interfaces $[48,49,50]$. The theory proved successful in capturing both surface tension and details of vapor-liquid molecular interfacial structures.

40 Gradient Theory simulations have been successfully performed for a wide variety of fluids: e.g., hydrocarbons and their mixtures [41, 51, 52], polar compounds and their mixtures $[53,54,55]$, polymer and polymer melts $[56,57,58]$, and vapor-liquid and liquid-liquid interfaces $[40,59,60,61,62]$.

The fidelity of Gradient Theory simulations depends on the fundamentally 45 consistent evaluation of the Helmholtz free energy density using an equation of state in meta-stable and unstable two-phase regimes of fluid densities, on vapor- 
liquid equilibrium phase properties, and on the direct correlation function of the homogeneous fluid. The development of equations of state has evolved in recent decades from simple cubic and virial equations to more complex equations

${ }_{50}$ of state. This development progressed from Beattie-Bridgeman and BenedictWebb-Rubin (BWR), to modified Benedict-Webb-Rubin (mBWR), and then to Helmholtz energy equations of state. It is well recognized, however, that widely applied highly-accurate multi-parameter equations of state are not valid inside two-phase regimes $[63,64]$. They generally produce unreasonable results such 55 as multiple Maxwell loops and excessively large values of pressures and related thermodynamic quantities. Hence, they may not be fully suitable for application in concert with Gradient Theory despite their demonstrated ability to accurately predict vapor and liquid phase saturation properties. This dilemma has long plagued Gradient Theory simulations which, as a consequence, have

60 been widely performed in combination with much less accurate cubic equations of state $[40,41,53,54,65,66]$. In this paper, one of the first studies is presented in which this dilemma is overcome. Gradient Theory is combined with a next-generation Helmholtz energy equation of state, developed by Lemmon [67]. In contrast to previous generations of equations of state, it exhibits a desirable

65 and fundamentally consistent behavior over the entire two-phase region due to its new functional form, recently presented by Lemmon and Jacobsen [63], and its incorporation of new experimental measurements in the critical region. Additional details on the significance of modeling terms in equations of state for critical and meta-stable two-phase regions have been documented by Tillner-

70 Roth [68]. This desirable behavior of the Helmholtz energy equation of state makes it fully suitable for high-fidelity Gradient Theory simulations. Reference simulations using this method are compared to corresponding results from cubic Peng-Robinson [69] equation of state, widely-used in combination with Gradient Theory as noted above, and modified Benedict-Webb-Rubin equation 75 of state [70].

The accuracy of Gradient Theory simulations also depends on the directcorrelation function of the homogeneous fluid which manifests in the influence 
parameter. It determines the molecular density profile gradient which balances local deviations of the species chemical potential from its equilibrium value. The

80 fundamental definition of the influence parameter, which formally includes the direct-correlation function, was independently derived by Bongiorno et al. [71] and Yang et al. [72]. Usually, however, required direct correlation functions are unavailable for the conditions and components of interest. Therefore, modeling expressions for the influence parameter have been developed using pure fluid

85 interface calculations [65, 73, 74, 75]. Such expressions demonstrated excellent results for pure fluid surface tension calculations and were also successfully used for modeling of multi-component vapor-liquid interface structures. These wellestablished modeling expressions generally utilize a reduced temperature as an independent parameter and, hence, are usually restricted to subcritical temperature regimes where facilitated two-phase pure-fluid surrogate models can be solved. This restriction may distort predictions for general multi-component interfaces where temperatures often exceed the critical temperature of vapor phase components. Then, the calculation of required pure component influence parameters is not well defined. In this paper, a more general modeling expression for the pure component influence parameter is introduced. Its development is motivated by its fundamental definition based on the direct correlation function of the homogeneous fluid and by performed high-fidelity simulations of interfacial density profiles. Using this model, excellent results were consistently observed for a wider range of relevant species. The model is shown to preserve the accuracy of previous temperature-dependent expressions, remains well-defined at supercritical temperatures, and is fully suitable for calculations of general multi-component two-phase interfaces.

\section{Materials and methods}

The development of equations of state has evolved over recent decades from simple cubic and virial equations to more complex equations of state such as Beattie-Bridgeman and Benedict-Webb-Rubin (BWR), modified Benedict- 
Webb-Rubin (mBWR), and Helmholtz energy equations of state. In this paper, a next-generation Helmholtz energy equation of state, originally developed by Lemmon [67], is utilized. In contrast to previous generations of equations of ${ }_{110}$ state, it exhibits a desirable and fundamentally consistent behavior over the entire two-phase region due to its new functional form, recently presented by Lemmon and Jacobsen [63], and its incorporation of new experimental measurements in the critical region. As a consequence, it facilitates fundamentally consistent calculations in meta-stable and unstable two-phase regimes of fluid densities [63]. This Helmholtz energy equation of state is applied in combination with vapor-liquid equilibrium and Gradient Theory and compared to frameworks based on cubic Peng-Robinson [69] and modified Benedict-WebbRubin equations of state [70].

\subsection{Helmholtz energy equation of state}

The applied Helmholtz energy equation of state for pure fluids is documented in detail in $[63,76,77]$ so that only a brief review of the specific equations, which are later used in the computational analysis with Gradient Theory, is provided here. A review of well-established cubic Peng-Robinson and modified BenedictWebb-Rubin equation of state is omitted and the reader is referred to the rich literature, cited above, in which such methods have been documented.

The Helmholtz energy equation of state utilizes reduced densities and temperatures as independent parameters. Its functional form reads

$$
\frac{A}{R T}=\alpha^{0}(\delta, \tau)+\alpha^{r}(\delta, \tau)
$$

with $T$ as the temperature, $R$ as the ideal gas constant, $\delta=\rho / \rho_{c}, \tau=T_{c} / T$, and with $A$ as the Helmholtz energy, split into a reduced ideal gas contribution ${ }_{130} \alpha^{0}$ and residual part $\alpha^{r}$ to account for intermolecular forces. The ideal gas Helmholtz energy results in [63]

$$
\alpha^{0}=\ln \delta-\ln \tau+\sum a_{k} \tau^{i_{k}}+\sum a_{k} \ln \left[1-\exp \left(-b_{k} \tau\right)\right]
$$

with $k$ as a running index for individual terms and with $a_{k}$ and $b_{k}$ as parameters 
discussed below. The residual Helmholtz energy reads [76]

$$
\begin{aligned}
\alpha^{r}= & \sum N_{k} \delta^{d_{k}} \tau^{t_{k}}+\sum N_{k} \delta^{d_{k}} \tau^{t_{k}} \exp \left(-\delta^{\ell_{k}}\right) \\
& +\sum N_{k} \delta^{d_{k}} \tau^{t_{k}} \exp \left(-\delta^{\ell_{k}}\right) \exp \left(-\tau^{m_{k}}\right)
\end{aligned}
$$

with fitting parameters $d_{k}, t_{k}, \ell_{k}$, and $m_{k}$ and coefficients $N_{k}$, discussed below. Then, the thermodynamic pressure can be derived as follows

$$
p=\rho R T\left(1+\delta\left(\frac{\partial \alpha^{r}}{\partial \delta}\right)_{\tau}\right)
$$

The compressibility factor is calculated according to

$$
Z=1+\delta\left(\frac{\partial \alpha^{r}}{\partial \delta}\right)_{\tau}
$$

The chemical potential $\mu$ is obtained by means of the fugacity coefficient $\phi$

$$
\phi=\exp \left[Z-1-\ln (Z)+\alpha^{r}\right]
$$

as follows

$$
\mu=\mu^{0}+R T \ln \phi
$$

with $\mu^{0}$ as the reference chemical potential. The required derivative of the residual Helmholtz energy in Eqs. (4) and (5) reads [63]

$$
\begin{aligned}
\delta \frac{\partial \alpha^{r}}{\partial \delta}= & \sum_{k=1}^{5} d_{k} N_{k} \delta^{d_{k}} \tau^{t_{k}}+\sum_{k=6}^{15} N_{k} \delta^{d_{k}} \tau^{t_{k}} \exp \left(-\delta^{\ell_{k}}\right)\left(d_{k}-\ell_{k} \delta^{\ell_{k}}\right) \\
& +\sum_{k=16}^{18} N_{k} \delta^{d_{k}} \tau^{t_{k}} \exp \left(-\delta^{\ell_{k}}\right) \exp \left(-\tau^{m_{k}}\right)\left(d_{k}-\ell_{k} \delta^{\ell_{k}}\right)
\end{aligned}
$$

The parameters for pentafluoroethane (R-125), applied in this study as an illustrative example, can be found in Lemmon and Jacobsen [63]. The listed parameters were obtained from a non-linear fit to available experimental data.

The generated equation demonstrated an estimated uncertainty of $0.1 \%$ in density and of $0.5 \%$ in the speed of sound over a wide range of gaseous and liquid states. Excessively large values of pressures and related thermodynamic properties in meta-stable and unstable regimes, typical of previous multi-parameter 
equations of state, have been eliminated. A desirable and fundamentally consistent behavior in the entire two-phase region was demonstrated due to its new functional form, presented by Lemmon and Jacobsen [63], and its incorporation of new experimental measurements in the critical region. Parameters for other species, which are analyzed later in this study, can be found elsewhere $[76,77]$.

\subsection{Gradient Theory for pure fluid interfaces}

According to Cahn and Hilliard [32], the equation for the Helmholtz free energy density suitable for molecular inhomogeneous regions is derived by expanding it as a truncated Taylor series in density

$$
F=\int\left[f_{0}\left(\rho_{M}\right)+\sum_{i, j} \frac{1}{2} \kappa_{i j} \nabla \rho_{M, i} \nabla \rho_{M, j}\right] d s
$$

with $F$ defined as the Helmholtz free energy, $f_{0}(\rho)$ the Helmholtz free energy density of a homogeneous fluid, $\rho_{M}$ the molar density, and $s$ the volume unit. 155 Note the different notations for the Helmholtz free energy in Eq. (9) in comparison to Eq. (1). Both of these notations are widely applied in the corresponding research communities and such standards are followed here. The influence parameter of the inhomogeneous fluid is denoted $\kappa$. It determines the molecular density profile gradient which balances local deviations of the species chemical potential from its equilibrium value. It is well recognized that the influence parameter can be assumed to be density-independent [42]. The equation for a planar interface is obtained by minimizing the Helmholtz free energy [40, 41]

$$
\sum_{i} \sum_{j} \frac{1}{2} \kappa_{i j} \frac{d \rho_{M, i}}{d z} \frac{d \rho_{M, j}}{d z}=\bar{\omega}\left(\rho_{M}\right)-\bar{\omega}_{s}
$$

with $z$ as the normal interface direction and $p_{s}=-\bar{\omega}_{s}$ as the equilibrium pressure. The grand thermodynamic potential energy density of a pure fluid is defined as

$$
\bar{\omega}\left(\rho_{M}\right)=f_{0}\left(\rho_{M}\right)-\rho_{M} \mu_{B}
$$

with $\mu_{B}$ as the equilibrium value of the species chemical potential. The local Helmholtz free energy density of a homogeneous fluid, calculated within the 
two-phase region, reads

$$
f_{0}\left(\rho_{M}\right)=\rho_{M} \mu\left(\rho_{M}\right)-p\left(\rho_{M}\right)
$$

with $p$ and $\mu$ as the homogeneous-fluid thermodynamic pressure and chemical potential, calculated using Eqs. (4) and (7), respectively. According to Gradient Theory, the surface tension for a pure fluid is obtained as follows

$$
\sigma=\int_{\rho_{V}}^{\rho_{L}} \sqrt{2 \kappa\left(\bar{\omega}\left(\rho_{M}\right)-\bar{\omega}_{s}\right)} d \rho
$$

with subscripts $V$ and $L$ denoting vapor and liquid saturation properties, respectively. Such saturation properties are obtained from vapor-liquid equilibrium calculations. A transformation from density-space to physical space allows the calculation of the interface profile

$$
z=z_{0}+\int_{\rho_{0}}^{\rho} \sqrt{\frac{\kappa}{2\left(\bar{\omega}\left(\rho_{M}\right)-\bar{\omega}_{s}\right)}} d \rho
$$

with $z_{0}$ as the reference spatial location at density $\rho_{0}$. The influence parameter $\kappa$ of the pure component is generally unknown. The fundamental definition of the influence parameter, which formally includes the direct-correlation function of the homogeneous fluid, was independently derived by Bongiorno et al. [71] 180 and Yang et al. [72] as

$$
\kappa(T, \rho, z)=\frac{k_{B} T}{6} \int z^{2} C_{0}(z, \rho) d z
$$

with $k_{B}$ as the Boltzmann constant, with $z$ as the spatial coordinate, and with $C_{0}$ as the direct correlation function of the homogeneous fluid at density $\rho$. Often, however, the required direct correlation functions for the evaluation of influence parameters are unavailable for the conditions and components of interest. Then, the pure component influence parameter is usually obtained from well-known surface tension data for pure fluids. Utilizing the assumption of density-independence of the influence parameter [42], it is commonly calculated as follows

$$
\kappa=\frac{1}{2}\left[\frac{\sigma_{\exp }}{\int_{\rho_{V}}^{\rho_{L}} \sqrt{\left(\bar{\omega}\left(\rho_{M}\right)-\bar{\omega}_{s}\right)} d \rho}\right]^{2}
$$


Modeling expressions for the influence parameter have been developed which utilized the temperature as an independent parameter in a fit to solutions of Eq. (16). Examples of those models have demonstrated excellent results for pure fluids [40, 41, 65, 66, 75]. It must be noted, however, that these modeling expressions are generally not fully suitable to calculate influence parameters in multi-component interfaces where temperatures often exceed the critical temperatures of vapor phase components. At such supercritical temperatures, the surface tension of the pure fluid diminishes and Eq. (16), along with their modeling expressions, become meaningless. Furthermore and as quantified below, previous generations of equations of state are generally invalid for calculations in the two-phase regime of fluid densities. Then, the widely-accepted application of Eq. (16) compensates for the inadequacies of those unsuitable equations of state in the fitting procedure to experimental data for pure fluid surface tension forces. As a consequence, however, the physical meaning of the calculated influence parameter becomes questionable since it serves as a tuning constant and does not necessarily represent a physical solution of Eq. (15). In this paper, a new fundamentally-based model for Eq. (15) is presented. It is shown to preserve the accuracy of previous temperature-dependent expressions, remains well-defined at supercritical temperatures, and is fully suitable for general multicomponent interface calculations.

\section{Results and Discussion}

Gradient Theory simulations of pure fluid interfaces are performed using Helmholtz energy equation of state. Illustrated on pentafluoroethane (R-125) as an example, obtained distributions of key thermodynamic properties in Gradient Theory across the vapor-liquid interfacial region are compared to results from Peng-Robinson and modified Benedict-Webb-Rubin equations of state. A generalized expression of the pure component influence parameter is presented. Its development is motivated by its fundamental definition based on the direct correlation function of the homogeneous fluid and by performed high-fidelity 
simulations of interfacial density profiles.

A detailed analysis of the interfacial thermodynamic properties within the vapor-liquid region is presented for pentafluoroethane. Its molecular weight, critical temperature, critical pressure, and critical density read $120.0214 \mathrm{~g} / \mathrm{mol}$, $339.173 \mathrm{~K}, 36.177$ bar, and $573.6 \mathrm{~kg} / \mathrm{m}^{3}$, respectively. Fundamental thermodynamic properties at the triple and normal boiling point are summarized in Table 1. The applied equation of state parameters in Eqs. (2), (3), and (8) can be found in Lemmon and Jacobsen [63]. The obtained equation was demonstrated to achieve an estimated uncertainty of overall $0.1 \%$ in density and of $0.5 \%$ in the speed of sound over a wide range of gaseous and liquid states. It exhibited a fundamentally consistent behavior in meta-stable and unstable two-phase regimes which is critical for accurate calculations of two-phase interfacial structures as demonstrated below. For orientation, Fig. 1 (left) presents a contour plot of densities in a classical temperature-pressure diagram for single-phase regimes. The saturation line separates the liquid and vapor phase and ends at the critical point. This also allows the definition of the compressed liquid, supercritical fluid, and ideal gas regime. Figure 1 (right) presents equilibrium densities of the vapor and liquid phase along the saturation line. For a pure fluid, the saturation curve separates the single-phase regime from the two-phase regime in which Gradient Theory is applied. In Gradient Theory, Eqs. (13) and (14) are solved in one-dimensional density space between the equilibrium vapor and liquid phase densities $\rho_{V}$ and $\rho_{L}$, respectively. To ensure that the presented results of this study are grid independent, a grid convergence study is performed. Figure 2 presents the calculated residuals of surface tension (left) and interface thickness (right) as a function of applied interface grid points to discretize the two-phase density space. Three different interface temperatures $\mathrm{T}=275 \mathrm{~K}, \mathrm{~T}=300 \mathrm{~K}$, and $\mathrm{T}=325 \mathrm{~K}$ have been investigated. The corresponding residual quantities are calculated from Eqs. (13) and (14). For these calculations, the influence parameter $\kappa$ has been set to an arbitrary value, here $\kappa=1$, since it has not been quantified yet. It is well recognized, however, that the influence parameter can be assumed to be density-independent [42]. Hence, it represents a constant pa- 
rameter in both equations. As a consequence, the grid convergence study is meaningful regardless of the specific value of the influence parameter. In this study, the algorithm automatically doubles the number of interface grid points until the pre-defined convergence criterion, here $\varepsilon=1.0 e-3$, is fulfilled for both quantities. In what follows, a grid convergence study has been performed for all presented quantities to guarantee grid-independent results.

Figure 3 compares computations of thermodynamic pressures for pentafluoroethane from Helmholtz energy equation of state using Eq. (4) with corresponding results from cubic Peng-Robinson (PR) and modified Benedict-WebbRubin (mBWR) equations of state in the two-phase regime of fluid densities. It is widely recognized that previous generations of multi-parameter equations of state generally produce unreasonable results in this regime such as multiple Maxwell loops and excessively large values of pressure (and related quantities) since they are generally invalid in meta-stable and unstable regions [63, 64]. The next-generation Helmholtz energy equation of state (EoS) applied here, however, has demonstrated desirable and fundamentally consistent behavior in 265 the entire two-phase region [63]. Figure 3 (a) illustrates that neither PR nor mBWR EoS succeed to qualitatively reproduce the reference pressure distribution obtained by Helmholtz energy EoS. Results from mBWR EoS exhibit multiple Maxwell loops. At somewhat higher temperatures (b), these multiple loops are eliminated and mBWR EoS succeeds, in contrast to PR EoS, to qualitatively reproduce the reference pressure distribution. At high temperatures (c), mBWR EoS and PR EoS both show qualitatively similar distributions which deviate from the reference solution. Results from mBWR EoS are in better agreement with Helmholtz energy EoS than corresponding results from PR EoS. The finding that neither PR nor mBWR EoS can consistently capture the qualitative distribution of key thermodynamic properties across the two-phase region is also confirmed in Fig. 4. It shows calculations of homogeneous-fluid chemical potentials, required in Gradient Theory by Eq. (12). Note that differences in chemical potentials, rather than their absolute values, carry physical significance. The results presented here are directly obtained from the respec- 
tive equation of state. It is important to realize that under many conditions and for different species, multi-parameter equations of state can indeed produce absurd results [63]. In summary, those equations may not be fully suitable for application in concert with Gradient Theory despite their proven ability to accurately predict saturation properties of vapor and liquid phases. It is worth mentioning that cubic equations of state have been widely used in previous studies in combination with Gradient Theory [40, 41, 53, 54, 65, 66]. However, the reference simulations using Helmholtz energy equation of state, presented in Figs. 3 and 4, have demonstrated complex distributions of key thermodynamic properties across the two-phase region. Due to the inherent lack of degrees of freedom in cubic expressions, these distributions cannot, by design, be reproduced from cubic equations of state. Hence, widely-acknowledged methods to improve calculations of saturation properties may not fully resolve all shortcomings of cubic equations of state for Gradient Theory simulations. These methods also require fitting data which is not necessarily available for the conditions of interest. However, cubic equations of state do not produce multiple Maxwell loops (like mBWR) and generally demonstrate a consistent behavior over a wide range of temperatures. It must be noted, however, that this behavior is rather a consequence of their inherent limit of degrees of freedom and does not necessarily reflect an underlying physical consistency.

In what follows, the fundamentally consistent high-accuracy Helmholtz energy equation of state is utilized to calculate the presented thermodynamic properties. A key quantity in Gradient Theory, next to the influence parameter, is the grand thermodynamical potential energy density defined in Eq. (11) and applied in Eqs. (13) and (14). Figure 5 presents its distribution in density space across the two-phase region for pentafluoroethane and for three different temperatures $\mathrm{T}=275 \mathrm{~K}, \mathrm{~T}=300 \mathrm{~K}$, and $\mathrm{T}=325 \mathrm{~K}$, respectively. The grand thermodynamical potential energy distribution diminishes with increasing temperature but it maintains its characteristic symmetrical bell shape, typical of a vapor-liquid system. It is important to realize that the fundamentally consistent calculation of this quantity establishes that solutions of Eq. (16) become physi- 
cally meaningful approximations for the solution of Eq. (15) for pure fluids. The development of a generalized expression of the influence parameter, fully suitable for multi-component interface simulations, is presented later. Note that the application of Eq. (16) in combination with previous generations of equations of state, generally invalid for calculations in the two-phase regime of fluid densities, calls the physical meaning of the calculated influence parameter into question since it then serves as a tuning constant in the fitting process to experimental data. The required surface tension $\sigma_{\text {exp }}$ represents the interfacial free energy force at different conditions along the saturation line of pure pentafluoroethane 320 and is, as such, considered a well-known fundamental thermodynamic quantity. A similar distribution as shown for the grand thermodynamical potential energy density is also obtained in Fig. 6 for the local spatial density gradient across the vapor-liquid interfacial region. The spatial density gradient is calculated as $\nabla \rho=\Delta \rho / \Delta z$ at each interface grid point. The relation between density and spatial dimension is established by Eq. (14). Figure 6 shows that interfacial density gradients diminish with increasing temperature. These calculations allow the evaluation of the true interfacial pressure distribution across the two-phase region. It follows from Eq. (12) for the pressure $p_{0}$ of a homogeneous fluid

$$
p_{0}=\rho \frac{\partial f_{0}}{\partial \rho}-f_{0}
$$

with $\partial f_{0} / \partial \rho=\mu$ as the chemical potential. Inserting the extended Helmholtz energy suitable for inhomogeneous fluid regions, compare Eq. (9), into the previous equation results in

$$
p=\rho \frac{\partial}{\partial \rho}\left(f_{0}+\frac{\kappa}{2}(\nabla \rho)^{2}\right)-\left(f_{0}+\frac{\kappa}{2}(\nabla \rho)^{2}\right)
$$

After rearranging, the resulting pressure can be split into the homogeneous-fluid and inhomogeneous-fluid contribution

$$
p=\underbrace{\rho \frac{\partial f_{0}}{\partial \rho}-f_{0}}_{p_{0}}+\rho \kappa \nabla^{2} \rho-\frac{\kappa}{2}(\nabla \rho)^{2}
$$

At equilibrium, the last two terms are equal and we arrive at $[32,38,39]$

$$
\frac{\partial p}{\partial z}=-\kappa \frac{\partial}{\partial z}(\nabla \rho)^{2}
$$


By integration and only strictly valid for equilibrium conditions, the pressure within the inhomogeneous fluid region is obtained

$$
p(z)=p_{s}-\kappa(\nabla \rho)^{2}
$$

with $p_{s}$ as the saturation pressure. Gradient Theory accommodates the calculation of the pressure distribution in the inhomogeneous vapor-liquid interface region. Such calculations are presented in Fig. 7. The inhomogeneous pressure 340 distribution across the two-phase regime shows a symmetric bell shape which exhibits negative pressures at lower temperatures. With increasing temperatures, the relative differences of the inhomogeneous pressure distribution to the saturation pressure diminishes. Note that the inhomogeneous pressure distribution approaches the homogeneous-fluid pressure solution only in the limit of the critical temperature where interfacial free energy forces diminish. The difference between both pressure distributions is also highlighted in Fig. 8, obtained from Helmholtz energy equation of state. As discussed above, this equation demonstrates, in contrast to previous multi-parameter equations of state, a fundamentally consistent behavior throughout the entire two-phase region, compare

350 Figs. 3 and 4. Figure 8 (a) shows the saturation dome of pentafluoroethane and the density-pressure pathway of an isotherm of $\mathrm{T}=315 \mathrm{~K}$. Outside of the two-phase region, the contribution of molecular density gradients to the local Helmholtz energy vanishes. As a consequence, results from Gradient Theory Helmholtz formulation and Helmholtz energy equation of state become indistinguishable. Inside the two-phase region, however, homogeneous-fluid calculations result in meta-stable and unstable fluid behavior, identified as $\partial p / \partial \rho>0$ (meta-stable) and $\partial p / \partial \rho<0$ (unstable), respectively. This definition reflects that unstable states are defined as conditions where infinitesimally small perturbations reduce the free energy. Then, no barrier exists which prevents the further evolution of such states toward the (stable) equilibrium. Therefore, the condition for stability is

$$
\left(\frac{\partial^{2} a}{\partial v^{2}}\right)_{T}>0
$$


with $v=1 / \rho$. We note that the homogeneous-fluid pressure is related to the free energy $a$ along an isotherm

$$
p=-\frac{\partial a}{\partial v}
$$

which leads to the distinctions between meta-stable and unstable regions of fluid densities presented above. Vapor and liquid meta-stable regions are identified as $\partial^{2} p / \partial \rho^{2}<0$ (vapor) and $\partial^{2} p / \partial \rho^{2}>0$ (liquid), respectively. Meta-stable regions are limited by the spinodal, identified as $\partial p / \partial \rho=0$. The liquid spinodal exists either at positive or negative pressure, compare Figs. 8 and 3, while the vapor spinodal is consistently located at positive pressures. These regions and spinodal are highlighted in Fig. 8. It is again noted that the homogeneous-fluid pressure distribution in the two-phase region, predicted by this Helmholtz energy equation of state, is fully consistent with those well-established definitions of meta-stable and unstable regimes of fluid densities. Previous generations of equations of state generally exhibit a behavior that is inconsistent with the physical behavior of fluids, compare Fig. 3. Unstable homogeneous-fluid states cannot be maintained in equilibrium. However, such states become realizable when they are stabilized by the balancing intermolecular forces which exist in inhomogeneous fluid regions. This balancing is accomplished by a local adjustment of the density gradient term, considered by Gradient Theory in Eq. (10). 380 Figure $8(\mathrm{~b})$ presents the isothermal $(\mathrm{T}=315 \mathrm{~K})$ homogeneous-fluid solution in a volume-pressure diagram. It illustrates the validity of the Maxwell-equal-arearule, exactly fulfilled by Helmholtz energy equation of state. The shaded regions $A_{1}$ and $A_{2}$ comprise the same area but with opposite signs. The volume has been calculated as $V=W /\left(\rho \cdot N_{A}\right)$ with $W$ as the molecular weight and $N_{A}$ as 385 the Avogadro number. Figure 8 (c) shows the transformation from density space into physical space in normal interface direction using Eq. (14). The interfacial density profile shows a characteristic shape similar to a tangent hyperbolic profile. The thickness of the interface can be extracted from this calculation as a characteristic length scale of a two-phase interface. It is also meaningful to transform the inhomogeneous pressure distribution into physical space as it 
reveals its relation to surface tension. From fundamental thermodynamics, it follows

$$
\sigma=\int \kappa(\nabla \rho)^{2} d z
$$

We note with Eq. (21), already derived,

$$
\sigma=\int\left(p_{s}-p(z)\right) d z
$$

This establishes that the shaded area, highlighted in Fig. 8 (c), is exactly equal to the net tangential force of the interface which manifests as the surface tension $\sigma$. The locations of the vapor and liquid spinodal are highlighted along the interfacial density profile. These locations span the region over which both the surface tension and the interfacial profile is largely determined. Hence, they demonstrate the significance of a highly accurate and fundamentally consistent behavior of the utilized equation of state in unstable two-phase regions for the computation of the interfacial properties.

Having established a framework fully suitable for analyzing vapor-liquid interfacial structures, a generalized expression for the influence parameter of pure components is introduced. Figure 9 (left) presents density profiles across the vapor-liquid interfacial region for pentafluoroethane at different reduced temperatures $\mathrm{T}_{r}=\mathrm{T} / \mathrm{T}_{c}$. With increasing temperatures, the interfacial thickness and vapor density increases and the liquid density decreases. The increase in vapor density is mainly due to the pressure increase which accompanies a temperature increase along the saturation line. The decrease in liquid den410 sity mainly results from increased temperature. The exponential increase of the vapor-liquid interfacial thickness with increasing temperature is highlighted in Fig. 9 (right). The relationship of the presented parameters density, spatial dimension, and temperature to the influence parameter $\kappa$ is shown by its fundamental definition, independently derived by Bongiorno et al. [71] and Yang ${ }_{415}$ et al. [72], presented in Eq. (15). Usually, direct correlation functions, required in Eq. (15), are unavailable for the conditions and species of interest. Therefore, modeling expressions for the influence parameter have been developed. 
Motivated by the apparent temperature dependence of Eq. (15), those have utilized the temperature as an independent parameter in a fit to solutions of ${ }_{420}$ Eq. (16). Examples of those models have demonstrated excellent results for pure fluids $[40,41,65,66,75]$. However, by definition, these approximations are restricted to subcritical temperatures for which Eq. (16) can be solved. At supercritical temperatures, surface tension forces diminish and Eq. (16) becomes meaningless. This restriction to subcritical temperatures, however, may distort

425 predictions for general multi-component interfaces where temperatures often exceed the critical temperature of vapor phase components. Then, no well-defined formula exists to calculate required pure component influence parameters. Note that this scenario describes the norm rather than the exception [48, 41, 78]. In this study, Eq. (15) is parameterized with the average density gradient as ${ }_{430}$ an independent variable. This parameter reflects explicitly the dependence of density and spatial dimension as two of the three parameters in Eq. (15) for the influence parameter. Furthermore, the temperature dependence is, as will be shown below, implicitly recovered by this parametrization. Utilizing a proper normalization with triple point properties $(t p)$, compare Table 1 , the normalized 435 density gradient reads

$$
\nabla \rho^{\star}=\frac{\nabla \rho-\left.\nabla \rho\right|_{t p}}{-\left.\nabla \rho\right|_{t p}}
$$

with the generic spatial gradient calculation of

$$
\nabla \rho=\frac{\rho_{L}-\rho_{V}}{\ell}
$$

Here, $\rho_{L}, \rho_{V}$, and $\ell$ denote the liquid density, vapor density, and interface thickness at the conditions of interest. Then, the new modeling expression for the influence parameter is established:

$$
\kappa(T, \rho, z)=c_{0}+c_{1} \nabla \rho^{\star} \approx \frac{k_{B} T}{6} \int z^{2} C_{0}(z, \rho) d z
$$

${ }_{440}$ with $c_{0}$ and $c_{1}$ as species-dependent coefficients and with $\nabla \rho^{\star}$ as the normalized density gradient, defined in Eq. (26). The validity of this approximation is demonstrated in Fig. 10. Figure 10 presents calculations of influence 
parameters for pentafluoroethane along the saturation line for each pressuretemperature pair as a function of the local normalized density gradient, cal445 culated by Eq. (26). The true physical influence parameter is calculated from meaningful approximations of its fundamental definition, compare Eq. (28) (and Eq. (15)) using Eq. (16). Note that this procedure is valid because a nextgeneration Helmholtz energy equation of state is applied here. As discussed above, it facilitates fundamentally consistent calculations over the entire two-

${ }_{450}$ phase regime and thereby establishes the physical meaning of the calculated grand thermodynamic potential energy density. As a consequence, Eq. (16) becomes a meaningful approximation for the right-hand-side of Eq. (28) for pure fluids. The analysis demonstrates a quasi-linear relationship between the influence parameter and the normalized density gradient, as postulated in Eq. (28).

455 A linear curve fit shows a maximal error to the true solution of less than $2 \%$. Applying this technique to a wider range of relevant species, similar relationships have been consistently observed. Figure 11 illustrates this physical complexity exemplarily for carbon dioxide. The corresponding linear curve fit is also in excellent agreement with the true solution and shows a maximal error of less than

${ }_{460} 3 \%$. It is important to note that the parametrization of the influence parameter with the normalized density gradient recovers the temperature dependence of previous expressions $[40,41,65,66]$. It is demonstrated in the following that the temperature dependence of the influence parameter, not explicitly included in the new generalized expression presented in Eq. (28) (or Eq. (26)), is implicitly

465 recovered. Figure 12 shows the normalized temperature as a function of the normalized density gradient for pentafluoroethane, carbon dioxide, propane, and decane as a representative set of relevant species. The normalized temperature is calculated as

$$
T^{\star}=\frac{T_{i}-T_{t p, i}}{T_{c, i}-T_{t p, i}}
$$

with $T_{c}$ and $T_{t p}$ as the critical temperature and triple point temperature of each species $i$, respectively. Figure 12 demonstrates the linear correlation between both quantities. Hence, the normalized density gradient approximation of the 
influence parameter, defined in Eq. (28), implicitly captures the well-recognized temperature dependence. It is important to realize that, in contrast to previous expressions explicit in reduced temperature, the validity of the presented

475 gradient expression is not limited to subcritical temperature regimes. The new generalized expression of Eq. (28) remains well-defined at supercritical temperatures and is fully suitable for calculations of general multi-component mixtures where temperatures often exceed the critical temperature of its vapor components. Then, the influence parameter for each component is calculated from the

480 species-specific normalized density gradient, computed according to corresponding vapor and liquid equilibrium properties and the thickness of the established interfacial region as prescribed by Eq. (26).

\section{Conclusions}

The fidelity of Gradient Theory simulations of pure fluid interfaces depends

485 on the accuracy of vapor-liquid equilibrium phase properties, influence parameters, and key thermodynamic quantities such as pressure and chemical potential distributions of equations of state across the entire two-phase regime. The development of equations of state has evolved from simple cubic and virial equations to more complex equations of state such as Beattie-Bridgeman and Benedict-

${ }_{490}$ Webb-Rubin, modified Benedict-Webb-Rubin, and Helmholtz energy equations of state. It is well recognized, however, that widely applied highly-accurate multi-parameter equations of state are not valid inside two-phase regimes. They generally produce unreasonable results such as multiple Maxwell loops and excessively large values of pressures, chemical potentials, and related thermodynamic quantities. Hence, they may not be fully suitable for application in concert with Gradient Theory despite their demonstrated ability to accurately predict vapor and liquid phase saturation properties. This dilemma has long plagued Gradient Theory simulations which, as a consequence, have been widely performed in combination with much less accurate cubic equations of state. In 500 this paper, one of the first studies was presented in which this dilemma was 
overcome. Gradient Theory was combined with a next-generation Helmholtz energy equation of state. In contrast to previous generations of multi-parameter equations of state, it exhibits a desirable and fundamentally consistent behavior over the entire two-phase region. This behavior makes it fully suitable for Gradient Theory simulations. Illustrated for pentafluoroethane, reference simulations using this method have been performed. They demonstrated the significance of highly accurate and fundamentally consistent calculations of unstable twophase regions for the computation of the interfacial properties. These reference simulations were compared to corresponding results from cubic Peng-Robinson equation of state and modified Benedict-Webb-Rubin equation of state. It was found that neither of those two methods succeeded to consistently capture the qualitative distribution of key thermodynamic properties in Gradient Theory across the two-phase region. Due to the inherent lack of degrees of freedom in cubic expressions, the demonstrated complex distributions cannot, by design, be reproduced from cubic equations of state. Hence, widely-acknowledged methods to improve the calculation of saturation properties may not fully resolve all limitations of cubic equations of state with respect to Gradient Theory simulations. These methods also require fitting data which is not necessarily available for the conditions of interest. However, cubic equations of state do not produce multiple Maxwell loops (like mBWR) and generally demonstrate a consistent behavior over a wide range of temperatures. It was noted, however, that this behavior is rather a consequence of their inherent limit of degrees of freedom and does not necessarily reflect an underlying physical consistency.

Performed analyses with the introduced framework informed the develop${ }_{525}$ ment of a generalized expression for pure component influence parameters. Motivated by its inherent temperature dependence, previous well-established modeling expressions often utilize temperature as an independent parameter in surrogate models for its computation. These formulas have demonstrated excellent results for pure fluids. Models explicit in temperature are usually restricted, however, to subcritical temperature regimes where two-phase purefluid surrogate models can be solved. This restriction may distort predictions 
for general multi-component interfaces where temperatures often exceed the critical temperature of vapor phase components. Then, the calculation of required pure component influence parameters is not well defined. In this paper, 535 a more physically-based model, which is not explicit in reduced temperature but instead utilizes an averaged normalized density gradient as the independent parameter, was introduced. It explicitly reflects the dependence of the influence parameter on density and spatial dimension, inherent in its fundamental definition. The normalized density gradient parameter was also shown to implicitly recover its well-recognized temperature dependence. Using this model for pentafluoroethane, carbon dioxide, pentane, and decane as a representative set of relevant species, excellent results were consistently observed. It was demonstrated that the new generalized expression for influence parameters preserves the accuracy of previous temperature-dependent expressions, remains well-defined at supercritical temperatures, and is fully suitable for general multicomponent two-phase interface simulations.

The invalidity of previous generations of highly-accurate multi-parameter equations of state in the two-phase regime and the utilization of influence parameters with questionable physical meaning has long blocked the pathway toward more fundamentally consistent multi-component Gradient Theory simulations. Future work will build on the presented generalized expression of the influence parameter and the application of highly-accurate and fundamentallyconsistent Helmholtz energy equation of state to demonstrate their significance to physically-based multi-component two-phase interface simulations.

\section{Acknowledgement}

This material is based upon work supported by the U.S. Department of Energy, Office of Science, Office of Basic Energy Sciences, Division of Chemical Sciences, Geosciences, and Biosciences under contract number DE-AC04-94AL85000. This research was performed at the Combustion Research Facility, Sandia National Laboratories, Livermore, California. I thank Dr. Eric W. Lem- 
mon of NIST, Boulder, for his assistance and suggestions during the development of the presented framework.

\section{References}

\section{References}

[1] G. M. Faeth, L.-P. Hsiang, P.-K. Wu, Structure and breakup properties of sprays, Int. J. Multiphase Flow 21 (1995) 99-127. doi:10.1016/ 0301-9322(95)00059-7.

[2] J. C. Lasheras, E. J. Hopfinger, Liquid jet instability and atomization in a coaxial gas stream, Annu. Rev. Fluid Mech. 32 (2000) 275-308. doi: 10.1146/annurev.fluid.32.1.275.

[3] A. Adamson, A. Gast, Physical Chemistry of Surfaces, Wiley-Interscience, 1997.

[4] J. Dann, Forces involved in the adhesive process: I. critical surface tensions of polymeric solids as determined with polar liquids, J. Colloid Interface Sci. 32 (1970) 302-320. doi:10.1016/0021-9797 (70)90054-8.

[5] C. Felsch, R.N. Dahms, B. Glodde, S. Vogel, S. Jerzembeck, N. Peters, H. Barths, T. Sloane, N. Wermuth, A.M. Lippert, An interactively coupled CFD-multi-zone approach to model HCCI combustion, Flow Turbul. Combust. 82 (2009) 621-641. doi:10.1007/s10494-009-9202-6.

[6] F. Perini, P.C. Miles, R.D. Reitz, A comprehensive modeling study of incylinder fluid flows in a high-swirl, light-duty optical diesel engine, Computers and Fluids 105 (2014) 113-124. doi:10.1016/j.compfluid.2014. 09.011.

[7] R.D. Reitz, G. Duraisamy, Review of high efficiency and clean reactivity controlled compression ignition (RCCI) combustion in internal combustion engines, Prog. Energy Combust. Sci.doi:10.1016/j.pecs.2014.05.003. 
[8] J. C. Oefelein, G. Lacaze, R. N. Dahms, A. Ruiz, A. Misdariis, Effects of real fluid thermodynamics in high-pressure fuel injection processes, SAE Intl. J. Engines 7 (2014) 1125-1136. doi:10.4271/2014-01-1429.

[9] D.A. Splitter, R.D. Reitz, Fuel reactivity effects on the efficiency and operational window of dual-fuel compression ignition engines, Fuel 118 (2014) 163-175. doi:10.1016/j.fuel.2013.10.045.

[10] J. C. Oefelein, R. N. Dahms, G. Lacaze, Detailed modeling and simulation of high-pressure fuel injection processes in diesel engines, SAE Intl. J. Engines 5 (2012) 1410-1419. doi:10.4271/2012-01-1258.

[11] Y. Li, Y. Chang, Y. Liu, M. Xie, T. Wang, L. Zhou, Parametric study and optimization of a RCCI (reactivity controlled compression ignition) engine fueled with methanol and diesel, Energy 65 (2014) 319-332. doi: $10.1016 / j$. energy . 2013.11.059.

[12] M. C. Drake, D. C. Haworth, Advanced gasoline engine development using optical diagnostics and numerical modeling, Proc. Combust. Inst. 31 (2007) 99-124. doi:10.1016/j.proci.2006.08.120.

[13] R. N. Dahms, C. Felsch, O. Röhl, N. Peters, Detailed chemistry flamelet modeling of mixed-mode combustion in spark-assisted HCCI engines, Proc. Combust. Inst. 33 (2011) 3023-3030. doi:10.1016/j proci.2010.08.005.

[14] B. Peterson, D.L. Reuss, V. Sick, On the ignition and flame development in a spray-guided direct-injection spark-ignition engine, Combust. Flame 161 (2014) 240-255. doi:10.1016/j.combustflame.2013.08.019.

[15] R. N. Dahms, N. Peters, D. W. Stanton, Z. Tan, J. Ewald, Pollutant formation modeling in natural gas SI engines using a level set based flamelet model, Int. J. Engine Res. 9 (2008) 1-14. doi:10.1243/ 14680874 JER02107. 
[16] B. Peterson, E. Baum, B. Böhm, A. Dreizler, Early flame propagation in a spark-ignition engine measured with quasi 4d-diagnostics, Proc. Combust. Inst. 35. doi:10.1016/j.proci.2014.05.131.

[17] R. N. Dahms, M. C. Drake, T. D. Fansler, R. O. Grover, A. S. Solomon, Detailed simulations of stratified ignition and combustion processes in a sprayguided gasoline engine using the SparkCIMM/G-equation modeling framework, SAE Int. J. Engines 5 (2012) 141-161. doi:10.4271/2012-01-0132.

[18] R. Stiel, J. Schorr, C. Krüger, A. Dreizler, B. Böhm, In-cylinder flow and fuel spray interactions in a stratified spray-guided gasoline engine investigated by high-speed laser imaging techniques, Flow Turbulence Combust. 91 (2013) 431-450. doi:10.1007/s10494-013-9500-x.

[19] R. N. Dahms, T. D. Fansler, M. C. Drake, T.-W. Kuo, A. M. Lippert, N. Peters, Modeling ignition phenomena in spray-guided spark-ignited engines, Proc. Combust. Inst. 32 (2009) 2743-2750. doi:10.1016/j.proci. 2008.05 .052$.

[20] C. Fajardo, V. Sick, Flow field assessment in a fired spray-guided sparkignition direct-injection engine based on UV particle image velocimetry with sub crank angle resolution, Proc. Combust. Inst. 31 (2007) 3023-3031. doi:10.1016/j.proci.2006.08.016.

[21] R. N. Dahms, M. C. Drake, T. D. Fansler, T.-W. Kuo, N. Peters, Understanding ignition processes in spray-guided gasoline engines using highspeed imaging and the extended spark-ignition model SparkCIMM. Part A: Spark channel processes and the turbulent flame front propagation, Combust. Flame 158 (2011) 2229-2244. doi:10.1016/j . combustflame.2011. 03.012 .

[22] R. N. Dahms, M. C. Drake, T. D. Fansler, T.-W. Kuo, N. Peters, Understanding ignition processes in spray-guided gasoline engines using highspeed imaging and the extended spark-ignition model SparkCIMM. Part B: 
Importance of molecular fuel properties in early flame front propagation, Combust. Flame 158 (2011) 2245-2260. doi:10.1016/j.combustflame. 2011.04 .003$.

[23] H. Barths, N. Peters, N. Brehm, A. Mack, M. Pfitzner, V. Smiljanovski, Simulation of pollutant formation in a gas-turbine combustor using unsteady flamelets, Symposium (International) on Combustion 27 (1998) 1841-1847. doi:10.1016/S0082-0784 (98) 80026-X.

[24] L.Y.M. Gicquel, G. Staffelbach, T. Poinsot, Large eddy simulations of gaseous flames in gas turbine combustion chambers, Prog. Energy Combust. Sci. 38 (2012) 782-817. doi:10.1016/j.pecs.2012.04.004.

[25] B. Franzelli, E. Riber, L.Y.M. Gicquel, T. Poinsot, Large eddy simulation of combustion instabilities in a lean partially premixed swirled flame, Combust. Flame 159 (2012) 621-637. doi:10.1016/j.combustflame.2011. 08.004 .

[26] W.P. Jones, A.J. Marquis, K. Vogiatzaki, Large-eddy simulation of spray combustion in a gas turbine combustor, Combust. Flame 161 (2014) 222239. doi:10.1016/j. combustflame.2013.07.016.

[27] S. Hermeth, G. Staffelbach, L.Y.M. Gicquel, T. Poinsot, LES evaluation of the effects of equivalence ratio fluctuations on the dynamic flame response in a real gas turbine combustion chamber, Proc. Combust. Inst. 34 (2013) 3165-3173. doi:10.1016/j.proci.2012.07.013.

[28] U. Stopper, W. Meier, R. Sadanandan, M. Stohr, M. Aigner, G. Bulat, Experimental study of industrial gas turbine flames including quantification of pressure influence on flow field, fuel/air premixing and flame shape, Combust. Flame 160 (2013) 2103-2118. doi:10.1016/j.combustflame. 2013.04 .005$.

[29] A. Laaksonen, V. Talanquer, D. Oxtoby, Nucleation: Measurements, the- 
ory, and atomospheric applications, Ann. Rev. Phys. Chem. 46 (1995) 489524. doi:10.1146/annurev.pc.46.100195.002421.

[30] P. Debenedetti, Metastable Liquids, Princeton University Press, 1996.

[31] P. Chaikin, T. Lubensky, Principles of Condensed Matter Physics, Cambridge University Press, 2000.

[32] J. W. Cahn, J. E. Hilliard, Free energy of a nonuniform system. I. Interfacial free energy, J. Chem. Phys. 28 (1958) 258-267. doi:10.1063/1.1744102.

[33] V. Mitlin, A new form of the Cahn-Hilliard equation: Applications to spinodal dewetting, J. Colloid Interface Sci. 281 (2005) 444-448. doi: $10.1016 / j \cdot j$ cis. 2004.08 .053$.

[34] J. Manin, M. Bardi, L. M. Pickett, R. N. Dahms, J. C. Oefelein, Microscopic investigation of the atomization and mixing processes of diesel sprays injected into high pressure and temperature environments, Fuel 134 (2014) 531-543. doi:10.1016/j.fuel.2014.05.060.

[35] W. Mayer, H. Tamura, Propellant injection in a liquid oxygen/gaseous hydrogen rocket engine, Journal of Propulsion and Power 12 (1996) 113711147. doi:10.2514/3.24154.

[36] R. N. Dahms, J. C. Oefelein, On the transition between two-phase and single-phase interface dynamics in multicomponent fluids at supercritical pressures, Phys. Fluids 25 (2013) 092103-1-24. doi:10.1063/1.4820346.

[37] R. N. Dahms, J. Manin, L. M. Pickett, J. C. Oefelein, Understanding highpressure gas-liquid interface phenomena in diesel engines, Proc. Combust. Inst. 34 (2013) 1667-1675. doi:10.1016/j.proci.2012.06.169.

[38] J. D. van der Waals, Square gradient model, Verhandel Konik Akad Weten Amsterdam 1 (1893) 8-15. 
[39] J. S. Rowlinson, Translation of J. D. van der Waals" "The thermodynamic theory of capillarity under the hypothesis of a continuous variation of density, J. Stat. Phys 20 (1979) 200-244.

[40] H. Lin, Y.-Y. Duan, Q. Min, Gradient Theory modeling of surface tension for pure fluids and binary mixtures, Fluid Phase Equil. 254 (2007) 75-90. doi:10.1016/j.fluid.2007.02.013.

[41] C. Miqueu, B. Mendiboure, A. Graciaa, J. Lachaise, Modeling of the surface tension of multicomponent mixtures with the gradient theory of fluid interfaces, Ind. Eng. Chem. Res. 44 (2005) 3321-3329. doi:10.1021/ ie0490861.

[42] B. F. McCoy, H. T. Davis, On the free energy theory of inhomogeneous fluids, Phys. Rev. A 20 (1979) 1201-1207. doi:10.1103/PhysRevA.20. 1201.

[43] V. Talanquer, D. Oxtoby, Dynamical density functional theory of gas-liquid nucleation, J. Chem. Phys. 100 (1994) 5190-5200. doi:10.1063/1.467183.

[44] M. Santra, S. Chakrabarty, B. Bagchi, Gas-liquid nucleation in a two dimensional system, J. Chem. Phys. 129 (2008) 234704-1-234704-6. doi: $10.1063 / 1.3037241$.

[45] D. Oxtoby, R. Evans, Nonclassical nucleation theory for the gas-liquid transition, J. Chem. Phys. 89 (1988) 7521-7530. doi:10.1063/1.455285.

[46] J. Cahn, Free energy of a nonuniform system. II. Thermodynamic basis, J. Chem. Phys. 30 (1959) 1121-1124. doi:10.1063/1.1730145.

[47] J. Cahn, J. Hilliard, Free energy of a nonuniform system. III. Nucleation in a two-component incompressible fluid, J. Chem. Phys. 31 (1959) 688-699. doi: $10.1063 / 1.1730447$.

[48] E. A. Müller, A. Mejia, Interfacial properties of selected binary mixtures containing n-alkanes, Fluid Phase Equilibria 282 (2009) 68-91. doi:10. 1016/j.fluid.2009.04.022. 
[49] A. Mejia, J. C. Pamies, D. Duque, H. Seguara, L. F. Vega, Phase and interface behaviors and type-I and type-V Lennard-Jones mixtures: Theory and simulations, J. Chem. Phys. 123 (2005) 1-10. doi:10.1063/1.1955529.

[50] C. Miqueu, J. M. Miguez, M. M. Pineiro, T. Lafitte, B. Mendiboure, Simultaneous application of the Gradient Theory and Monte Carlo molecular simulation for the investigation of methane/water interfacial properties, J. Phys. Chem. B 115 (2011) 9618-9625. doi:10.1021/jp202276k.

[51] S. Liu, D. Fu, J. Lu, Investigation of bulk and interfacial properties for nitrogen and light hydrocarbon binary mixtures by perturbed-chain statistical associating fluid theory combined with density-gradient theory, Ind. Eng. Chem. Res. 48 (2009) 10734-10739. doi:10.1021/ie9007437.

[52] J. Dechoz, C. Roze, Surface tension measurement of fuels and alkanes at high pressure under different atmospheres, Appl. Surf. Sci. 229 (2004) 175182. doi:10.1016/j.apsusc.2004.01.057.

[53] A. Mejia, H. Segura, L. Vega, J. Wisniak, Simulataneous prediction of interfacial tension and phase equilibria in binary mixtures. an approach based on cubic equations of state with improved mixing rules, Fluid Phase Equilib. 227 (2005) 225-238. doi:10.1016/j.fluid.2004.10.024.

[54] K. A. G. Schmidt, G. K. Folas, B. Kvamme, Calculation of the interfacial tension of methane-water system with the linear gradient theory, Fluid Phase Equil. 261 (2007) 230-237. doi:10.1016/j.fluid.2007.07.045.

[55] V. Vins, B. Plankova, J. Hruby, Surface tension of binary mixtures including polar components modeled by the density gradient theory combined with the PC-SAFT equation of state, Int. J. Thermophys. 34 (2013) 792812. doi: $10.1007 / \mathrm{s} 10765-012-1207-z$.

[56] G. Dee, B. Sauer, The molecular weight and temperature dependence of polymer surface tension: Comparison of experiment with interface gra- 
dient theory, J. Colloid Interface Sci. 152 (1992) 85-103. doi:10.1016/ 0021-9797 (92)90010-J.

[57] M. Oliveira, J. Coutinho, A. Queimada, Surface tension of esters from a combination of the gradient theory with the CPA EoS, Fluid Phase Equilib. 303 (2011) 56-61. doi:10.1016/j.fluid.2011.01.015.

[58] B. Sauer, G. Dee, Studies of polymer, copolymer, and associating liquids by melt surface tension methods and Cahn-Hilliard density-gradient-theory, J. Colloid Interface Sci. 162 (1994) 25-35. doi:10.1006/jcis.1994.1004.

[59] V. Mitlin, Gradient theory for structural forces in thin fluid fils near the critical point, J. Colloid Interface Sci. 278 (2004) 173-183. doi:10.1016/ j.jcis. 2004.05 .022 .

[60] O. G. Nino-Amezquita, S. Enders, P. T. Jaeger, R. Eggers, Measurement and prediction of interfacial tension of binary mixtures, Ind. Eng. Chem. Res. 49 (2010) 592-601. doi:10.1021/ie901209z.

[61] R. N. Dahms, J. C. Oefelein, Non-equilibrium gas-liquid interface dynamics in high-pressure liquid injection systems, Proc. Combust. Inst. 35. doi: 10.1016/j.proci.2014.05.155.

[62] W. Qu, C. Yang, D. Li, A gradient theory approach to line tension of liquid-liquid-fluid systems, Colloids Surf. A 144 (1998) 275-285. doi: S0927-7757 (98) 00541-X.

[63] E. Lemmon, R. Jacobsen, A new functional form and new fitting techniques for equations of state with application to pentafluoroethane, J. Phys. Chem. Ref. Data 34 (2005) 69-108. doi:10.1063/1.1797813.

[64] R. Span, W. Wagner, E. Lemmon, R. Jacobsen, Multiparameter equations of state - recent trends and future challenges, Fluid Phase Equilib. 183-184 (2001) 1-20. doi:10.1016/S0378-3812(01) 00416-2. 
[65] C. Miqueu, B. Mendiboure, A. Graciaa, J. Lachaise, Modelling of the surface tension of pure components with the gradient theory of fluid interfaces: a simple and accuracte expression for the influence parameters, Fluid Phase Equilib. 207 (2003) 225-246. doi:10.1016/S0378-3812(03)00028-1.

[66] Y.-X. Zuo, E. H. Stenby, A linear gradient theory model for calculating, interfacial tensions of mixtures, J. Colloid Interface Sci. 182 (1996) 126132. doi:10.1006/jcis.1996. 0443.

[67] E. Lemmon, A generalized model for the prediction of the thermodynamic properties of mixtures including vapor-liquid equilibrium, Ph.D. thesis, University of Idaho (1996).

[68] R. Tillner-Roth, Fundamental Equations of State, Shaker Verlag, Aachen, Germany, 1998.

[69] D.-Y. Peng, D. Robinson, A new two-constant equation of state, Ind. Eng. Chem. Fundam. 15 (1976) 59-64. doi:10.1021/i160057a011.

[70] R. T. Jacobsen, R. B. Stewart, Thermodynamic properties of nitrogen including liquid and vapor phases from $63 \mathrm{k}$ to $2000 \mathrm{k}$ with pressures to 10,000 bar, J. Phys. Chem. Ref. Data 2 (1973) 757-922.

[71] V. Bongiorno, L. Scriven, H. Davis, Molecular theory of fluid interfaces, J. Colloid Interface Sci. 57 (1976) 462-475. doi:10.1016/0021-9797(76) 90225-3.

[72] A. Yang, P. F. III, J. Gibbs, Molecular theory of surface tension, J. Chem. Phys. 64 (1976) 3732-3747. doi:10.1063/1.432687.

[73] J. Perez-Lopez, J. Puig, M. Leiva, Gradient theory of surface tension for pure fluids: Effects of molecular shape and polarity, Physica A 172 (1991) 309-319. doi:10.1016/0378-4371(91)90385-P.

[74] J. Perez-Lopez, L. Gonzalez-Ortiz, M. Leiva, J. Puig, Estimation of surface tension of pure fluids using the gradient theory, AIChE J. 38 (1992) 753760. doi:10.1002/aic.690380512. 
[75] B. Breure, C. Peters, Modeling of the surface tension of pure components and mixtures using the density gradient theory combined with a theoretically derived influence parameter correlation, Fluid Phase Equilib. 334 (2012) 189-196. doi:10.1016/j.fluid.2012.08.010.

[76] E. Lemmon, R. Tillner-Roth, A Helmholtz energy equation of state for calculating the thermodynamic properties of fluid mixtures, Fluid Phase Equilib. 165 (1999) 1-21. doi:10.1016/S0378-3812(99)00262-9.

[77] E. Lemmon, M. Huber, M. McLinden, NIST standard reference database 23: Reference fluid thermodynamic and transport properties - Refprop, version 9.1, National Institute of Standard and Technology, Standard Reference Data Program, Gaithersburg.

[78] C. Miqueu, B. Mendiboure, C. Graciaa, J. Lachaise, Modelling of the surface tension of binary and ternary mixtures with the gradient theory of fluid interfaces, Fluid Phase Equilibria 218 (2004) 189-203. doi: 10.1016/j.fluid.2003.12.008. 


\section{Tables}

Triple Point Normal Boiling Point

\begin{tabular}{lcc}
\hline Temperature $(\mathrm{K})$ & 172.52 & 225.06 \\
Pressure (bar) & 0.02914 & 1.0132 \\
Liquid density $\left(\mathrm{kg} / \mathrm{m}^{3}\right)$ & 1690.680 & 1513.594 \\
Vapor density $\left(\mathrm{kg} / \mathrm{m}^{3}\right)$ & 0.2446 & 6.7895
\end{tabular}

Table 1: Summary of temperatures, pressures, and liquid and vapor densities at the triple point and normal boiling point for pentafluoroethane (R-125), calculated from Helmholtz energy equation of state. 


\section{Figures}
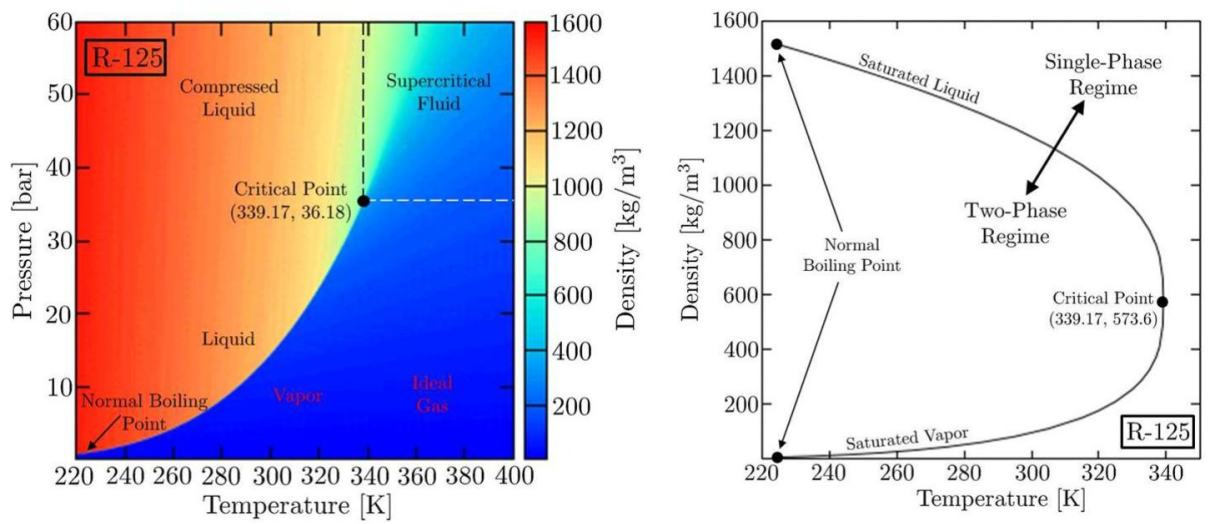

Figure 1: (Left) Density contour plot for pentafluoroethane (R-125) in a single-phase pressuretemperature diagram. (Right) Liquid and vapor densities along the saturation line between the normal boiling point and the critical point. These density values separate the single-phase and two-phase regime and serve as boundary conditions for Eqs. (13) and (14). 

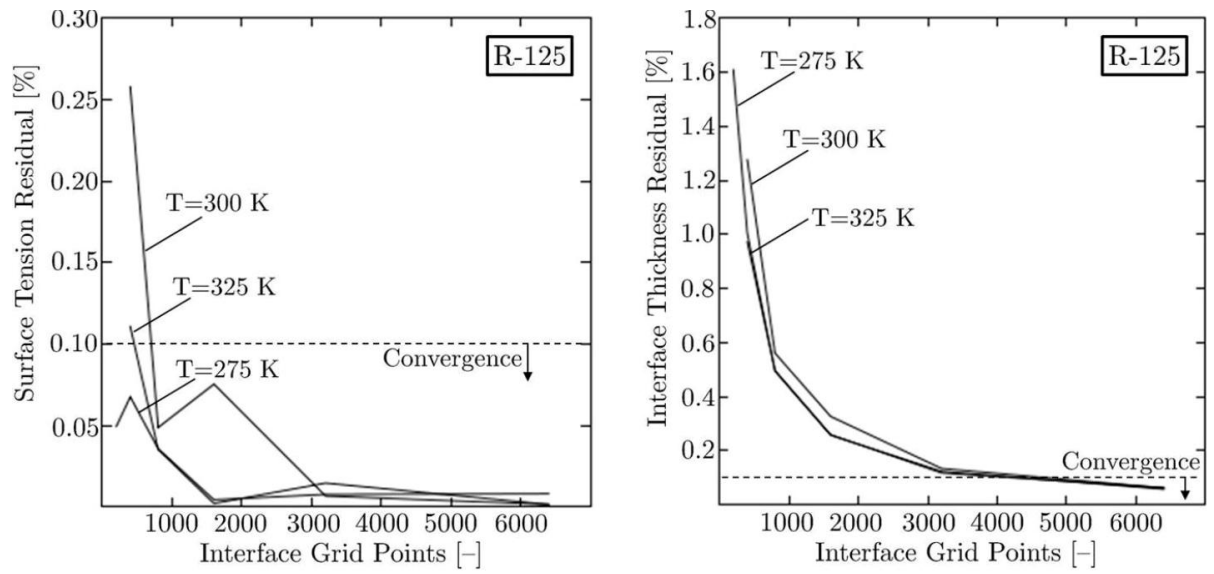

Figure 2: Calculated residuals of surface tension (left) and interface thickness (right) of pentafluoroethane as a function of the applied number of interface grid points for three different interface temperature. The algorithm adds interface grid points until the defined convergence criterion, here $\varepsilon=1.0 \mathrm{e}-3$, is fulfilled. 

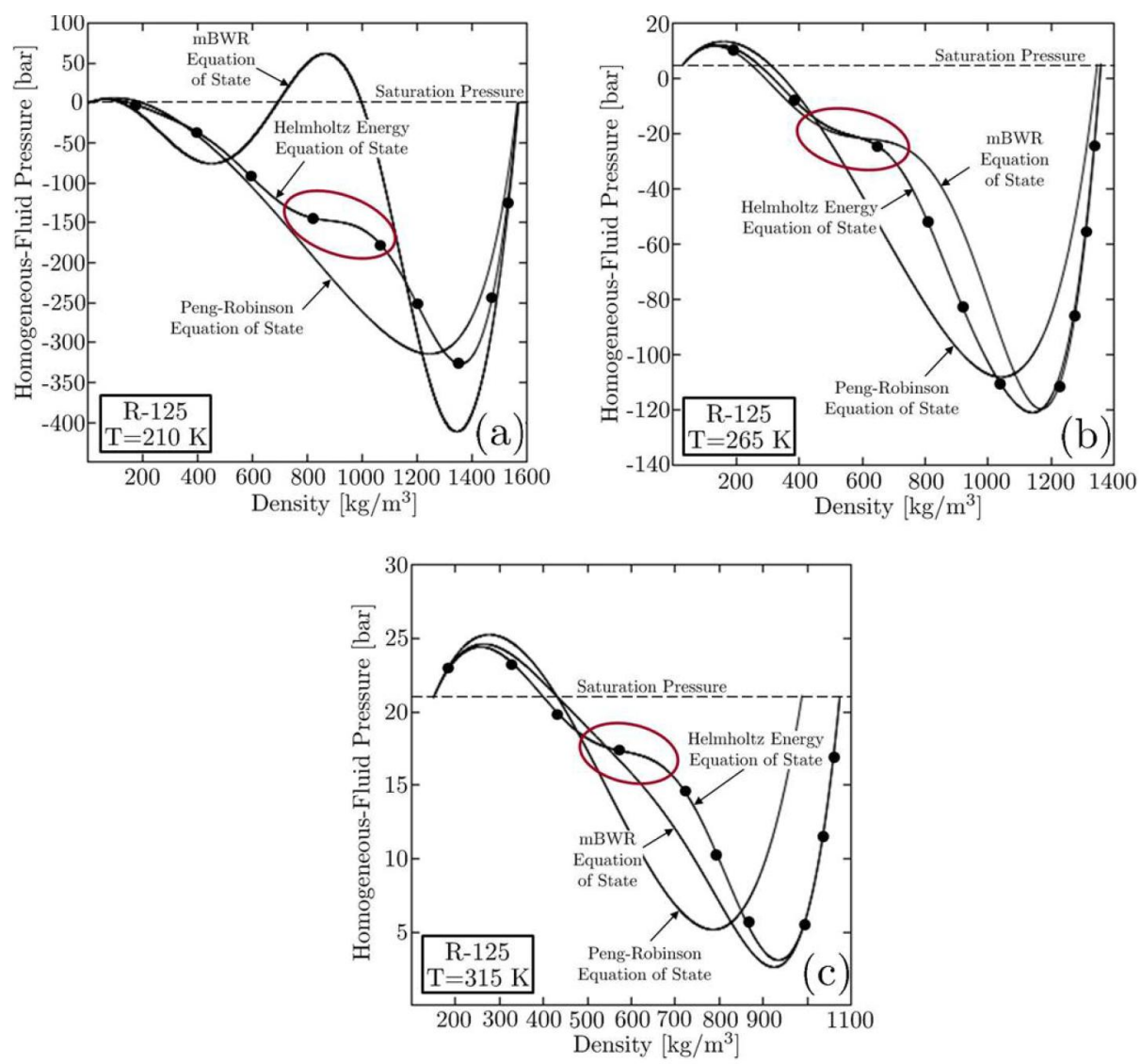

Figure 3: Comparison of homogeneous-fluid pressure calculations in the two-phase region using Peng-Robinson, modified Benedict-Webb-Rubin (mBWR), and Helmholtz energy equation of state (Eq. (4)) for pentafluoroethane at three different temperatures (a) $\mathrm{T}=210 \mathrm{~K}$, (b) $\mathrm{T}=265$ $\mathrm{K}$, and (c) $\mathrm{T}=315 \mathrm{~K}$. The highlighted region illustrates a consistent pattern of the Helmholtz energy reference simulation which cannot be consistently reproduced by either PR or mBWR equation of state. 

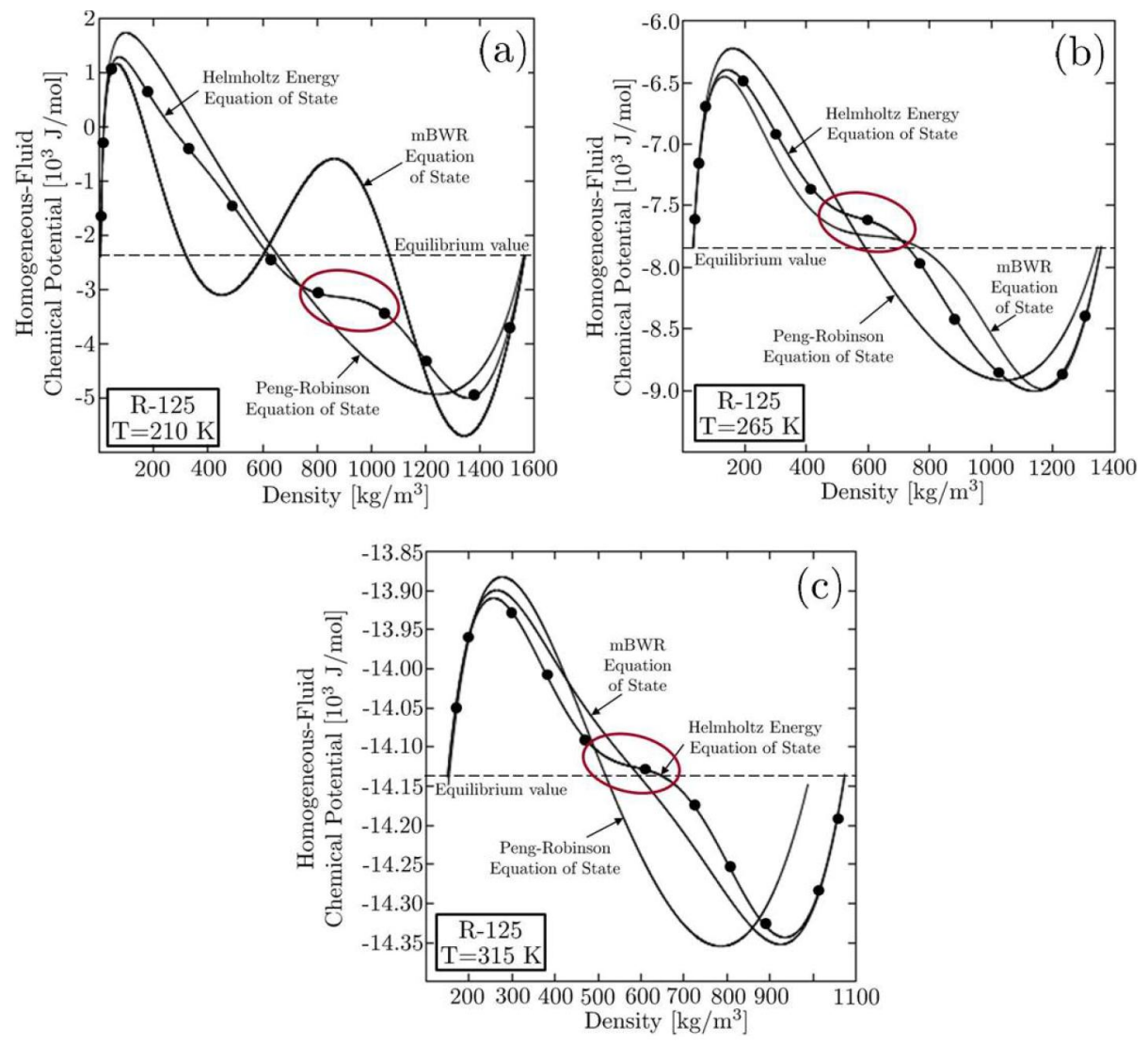

Figure 4: Comparison of homogeneous-fluid chemical potential calculations in the two-phase region using Peng-Robinson, modified Benedict-Webb-Rubin (mBWR), and Helmholtz energy equation of state for pentafluoroethane at three different temperatures (a) $\mathrm{T}=210 \mathrm{~K}$, (b) $\mathrm{T}=265 \mathrm{~K}$, and (c) $\mathrm{T}=315 \mathrm{~K}$. Note that differences in chemical potential, rather than their absolute values, carry physical significance. The highlighted region illustrates a consistent pattern of the Helmholtz energy reference simulation which cannot be consistently reproduced by either PR or mBWR equation of state. 


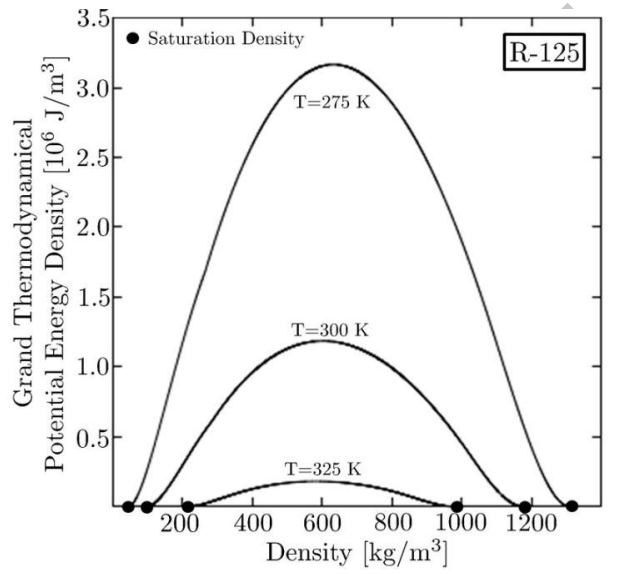

Figure 5: Calculations of grand thermodynamical potential energy density in the two-phase region from Eq. (11) using Helmholtz energy equation of state for pentafluoroethane at three different temperatures $\mathrm{T}=275 \mathrm{~K}, \mathrm{~T}=300 \mathrm{~K}$, and $\mathrm{T}=325 \mathrm{~K}$, respectively. The grand thermodynamical potential energy distribution diminishes with increasing temperature but maintains its characteristic symmetric bell shape. Filled circles mark the respective saturation densities. 


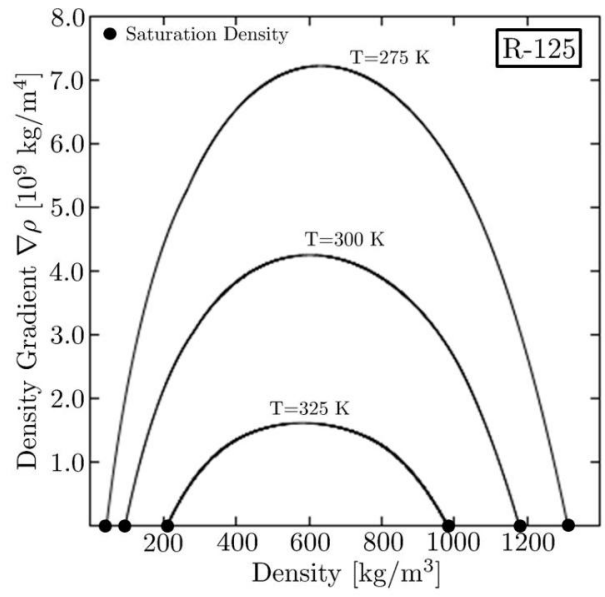

Figure 6: Calculations of spatial density gradients along the interface density profile in the two-phase region using Helmholtz energy equation of state for pentafluoroethane at three different temperatures $\mathrm{T}=275 \mathrm{~K}, \mathrm{~T}=300 \mathrm{~K}$, and $\mathrm{T}=325 \mathrm{~K}$, respectively. The density gradient distribution diminishes with increasing temperature but maintains its characteristic symmetric bell shape. Filled circles mark the respective saturation densities. 


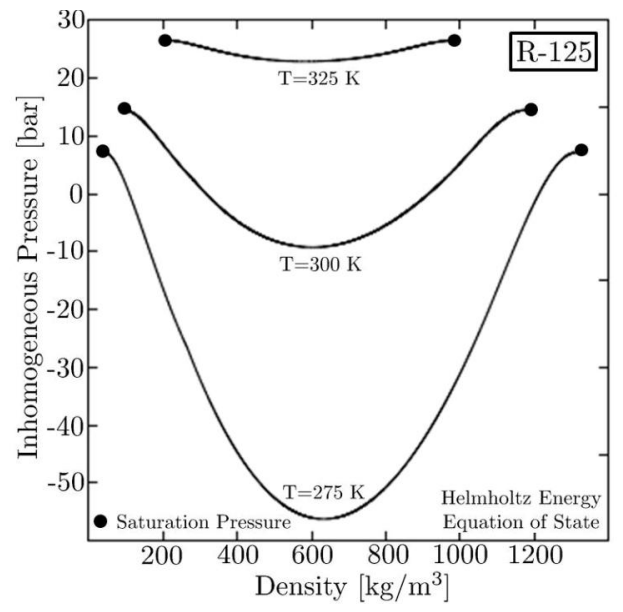

Figure 7: Calculations of the true inhomogeneous pressure distribution in the two-phase region from Eq. (21) using Gradient Theory and Helmholtz energy equation of state for pentafluoroethane at three different temperatures $\mathrm{T}=275 \mathrm{~K}, \mathrm{~T}=300 \mathrm{~K}$, and $\mathrm{T}=325 \mathrm{~K}$, respectively. Filled circles mark the respective saturation pressures. 

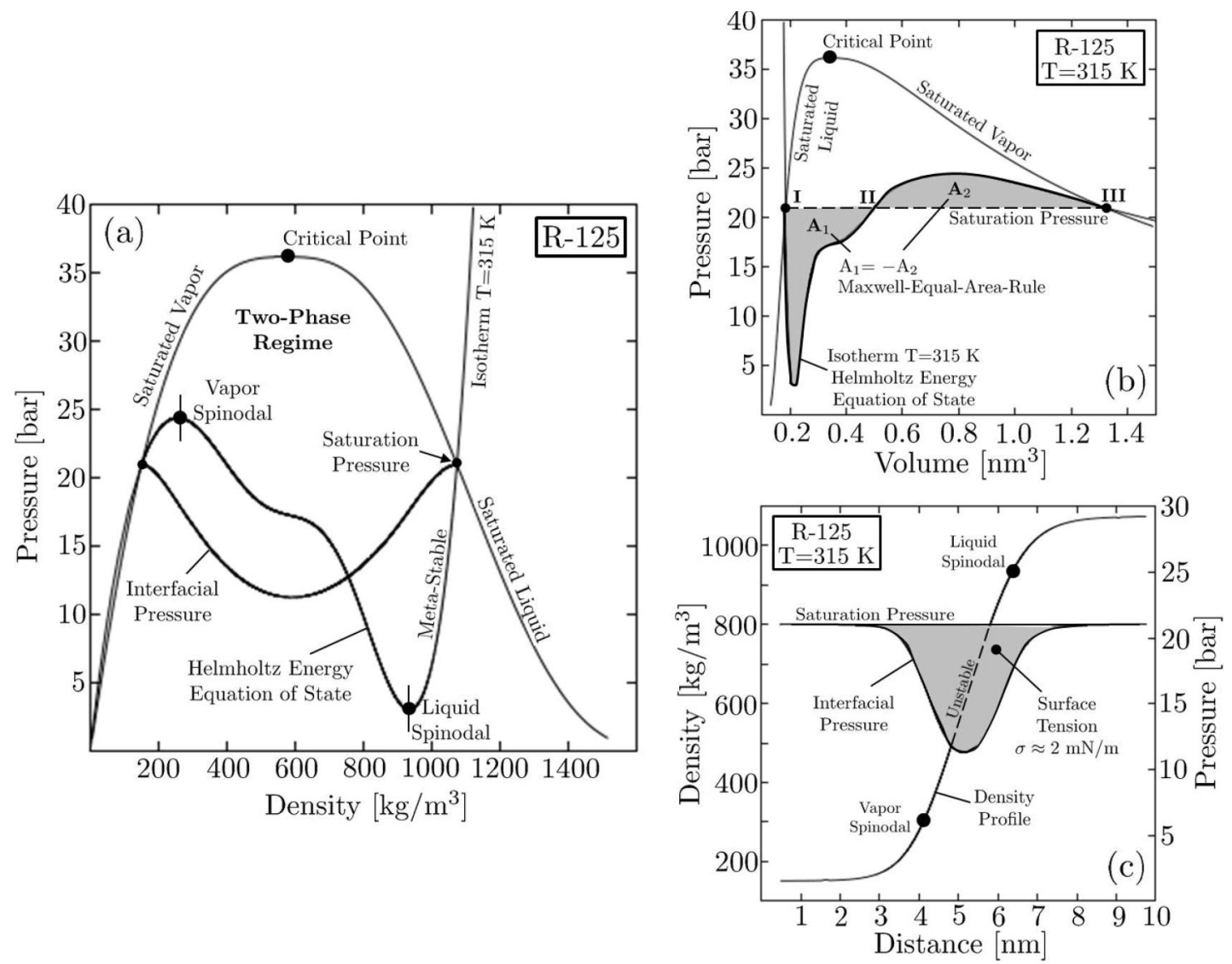

Figure 8: (a) Calculation of the saturation dome and the density-pressure envelope for pentafluoroethane along the isotherm $\mathrm{T}=315 \mathrm{~K}$. Note the qualitative differences in the twophase region between the pressure distribution, obtained from Helmholtz energy equation of state and calculated from Eq. (21). (b) The volume-pressure pathway demonstrates the validity of the Maxwell-equal-area-rule, exactly fulfilled by the equation of state. The volume has been calculated as $V=W /\left(\rho \cdot N_{A}\right)$ with $W$ as the molecular weight and $N_{A}$ as the Avogadro number. (c) Calculations show the interfacial density profile, the interfacial pressure distribution which is exactly related to the surface tension $\sigma$, and the locations of the vapor and liquid spinodal. These locations span the region over which both the surface tension and the interfacial profile is largely determined. Hence, they demonstrate the significance of fundamentally consistent calculations of unstable two-phase regions for the computation of the interfacial properties. 

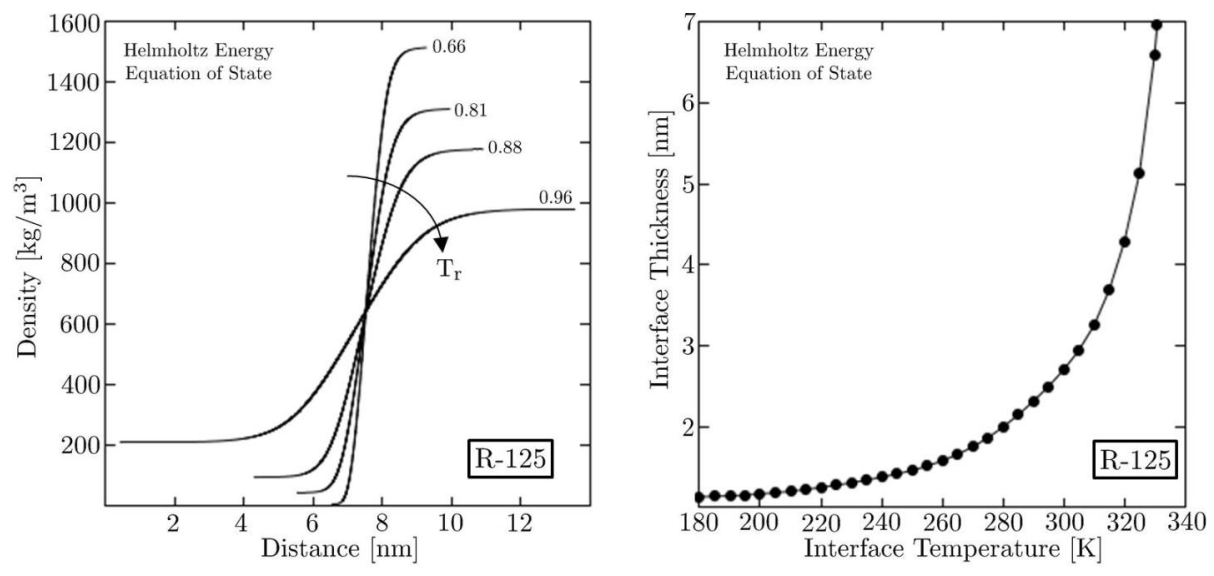

Figure 9: (Left) Calculations of interface density profiles using Helmholtz energy equation of state for pentafluoroethane at different reduced temperatures $\mathrm{T}_{r}=\mathrm{T} / \mathrm{T}_{c}$. (Right) Calculated interface thicknesses as a function of the interface temperature. 


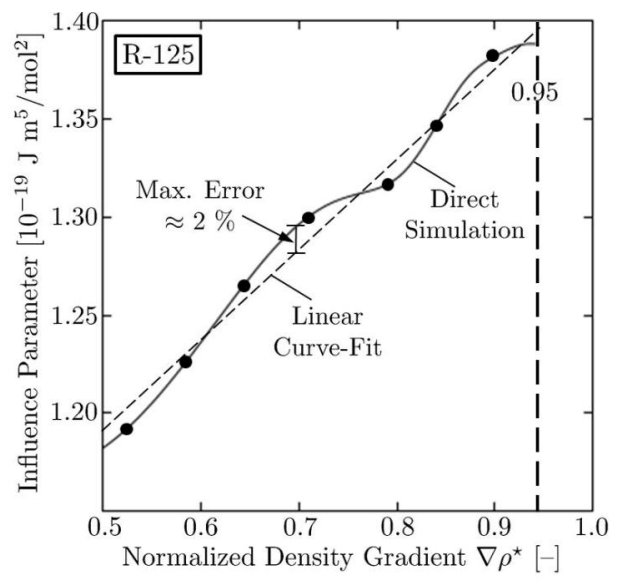

Figure 10: Calculations of influence parameters for pentafluoroethane, compare Eq. (28), as a function of the normalized density gradient, defined in Eq. (26). A linear curve fit approximates the true relation with a maximal error of $\varepsilon \approx 2 \%$. Calculations of near-critical quantities $\left(\nabla \rho^{\star}>0.95\right)$ have not been attempted. 


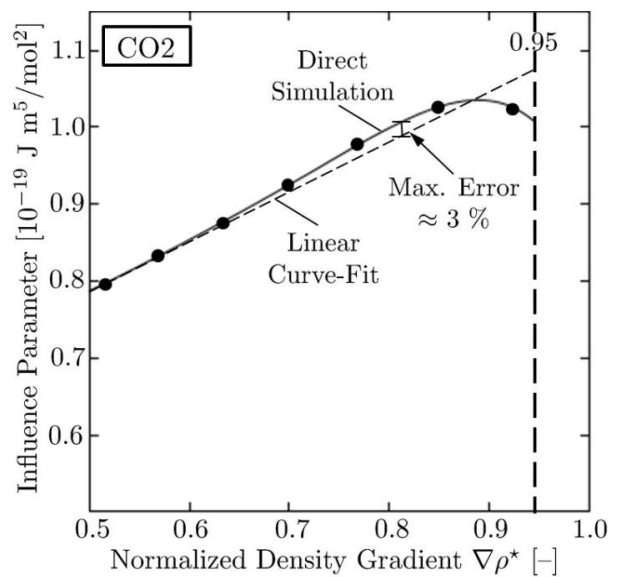

Figure 11: Calculations of influence parameters for carbon dioxide, compare Eq. (28), as a function of the normalized density gradient, defined in Eq. (26). A linear curve fit approximates the true relation with a maximal error of $\varepsilon \approx 3 \%$. Calculations of near-critical quantities $\left(\nabla \rho^{\star}>0.95\right)$ have not been attempted. 


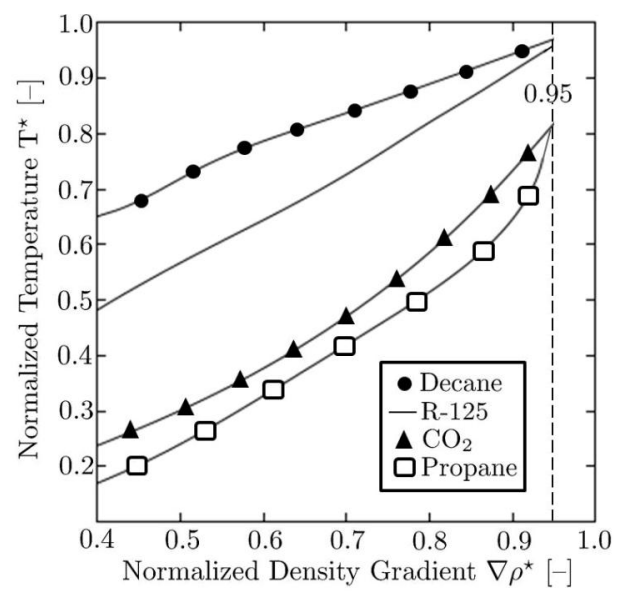

Figure 12: Calculations of normalized temperatures as a function of the normalized density gradient, defined by Eqs. (29) and (26), respectively, for decane, pentafluoroethane, carbon dioxide, and propane as a representative set of relevant species. The calculations demonstrate a linear relation between both normalized variables. Calculations of near-critical quantities $\left(\nabla \rho^{\star}>0.95\right)$ have not been attempted. 

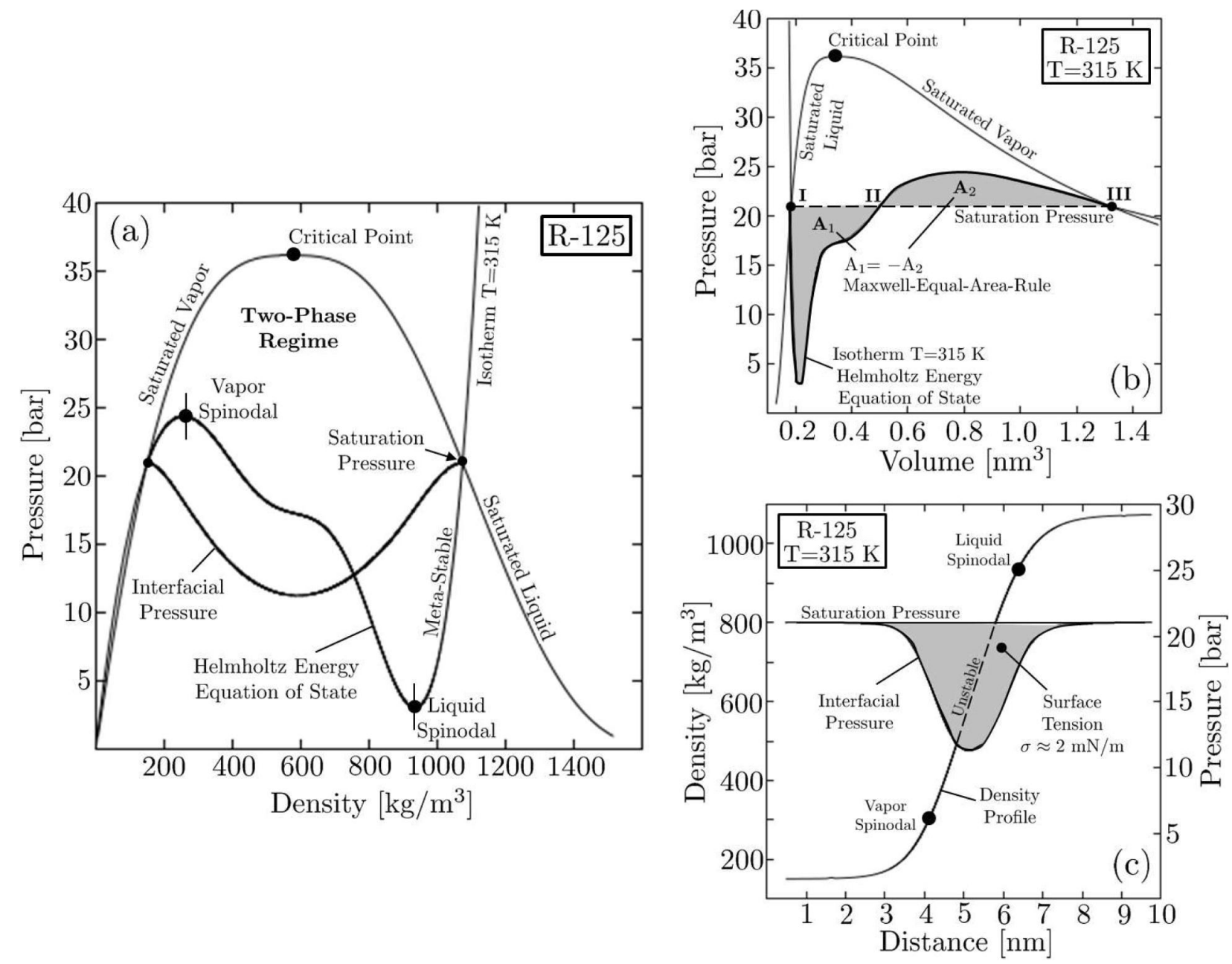

(a) Calculation of the saturation dome and the density-pressure envelope for pentafluoroethane along the isotherm $T=315 \mathrm{~K}$. Note the qualitative differences in the two-phase region between the pressure distribution, obtained from NIST Helmholtz energy equation of state and from Gradient Theory. (b) The volume-pressure pathway demonstrates the validity of the Maxwell-equal-area-rule, exactly fulfilled by the applied equation of state. (c) Calculations show the interfacial density profile, the interfacial pressure distribution which is exactly related to the surface tension, and the locations of the vapor and liquid spinodal. These locations span the region over which both the surface tension and the interfacial profile is largely determined. Hence, they demonstrate the significance of fundamentally consistent calculations of unstable twophase regions, achieved here, for the computation of the interfacial properties. 